\title{
Second Harmonic Generation with a Fractional Order of Periodical Poling
}

\author{
V.V. Dudelev ${ }^{1}$, K.A. Fedorova ${ }^{2}$, D.V. Chistyakov ${ }^{3}$, K.K. Soboleva ${ }^{4}$, V.E. Bugrov ${ }^{3}$, E.U. Rafailov ${ }^{5}$, G.S. Sokolovskii ${ }^{1}$ \\ ${ }^{1}$ Ioffe Institute, Saint Petersburg, Russia \\ ${ }^{2}$ Philipps University of Marburg, Marburg, Germany \\ ${ }^{3}$ ITMO University, Saint Petersburg, Russia \\ ${ }^{4}$ Peter the Great St. Petersburg Polytechnic University, Saint Petersburg, Russia \\ ${ }^{5}$ Aston University, Birmingham, UK \\ gs@mail.ioffe.ru
}

\begin{abstract}
We demonstrate second harmonic generation in a diode-pumped periodically-poled lithium niobate crystal with a fractional poling period.
\end{abstract}

Keywords - second harmonic generation, nonlinear crystal, periodical poling

Frequency doubling of the infrared (IR) laser diode radiation seems to be one of the most interesting aproaches for visible laser generation especially in wavelength ranges unreachable for 'direct' diodes [1]. The utilization of a waveguided periodically-poled (PP) [2] crystal can provide high efficiency of the second harmonic generation (SHG) above 50\% [3] even with spatially-imperfect laser diode radiation. However, despite the demonstrated higher-order and multimode phase matching [4] techniques, the achieved to date SHG tunabilty does not match extremely broad tunability of IR semiconductor lasers.

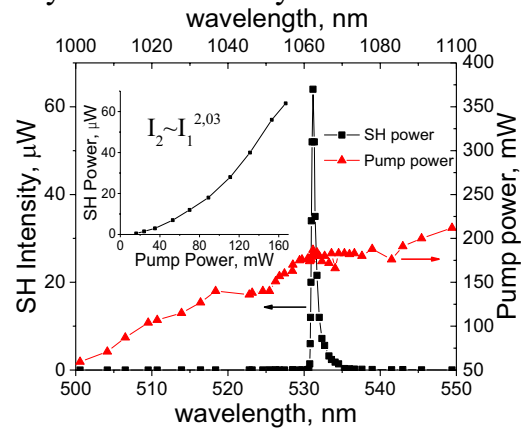

Fig.1. SHG power in the $1^{\text {st }}$ order of periodical poling (black squares) and pump power (red triangles) vs wavelength. Inset shows SHG power vs pump power.

With conventional periodical poling, its wave vector compensates the mismatch of the wave vectors of photons of SHG and fundamental waves. With the recently proposed fractional order of periodical poling [5], the mismatch of the wave vectors of $m$ pairs of fundamental photons and $m$ SHG photons is compensated by $p$ wave vectors of the PP lattice in a nonlinear crystal. This may result not only in the 'red' shift of the SHG wavelength, but also in $p / m$ extension of the SHG tuning range when applied with the multimode phase matching technique.

For the experimental demonstration of the SHG in the fractional order, we used a bulk lithium niobate crystal periodically poled for SHG at a wavelength of $530 \mathrm{~nm}$. The crystal was pumped by two broadly tunable IR laser diodes covering wavelength ranges corresponding to the $1^{\text {st }}$ and $1 / 2-$ order of periodical poling: $1000-1100 \mathrm{~nm}$ and $1260-1380 \mathrm{~nm}$, correspondingly. The experimental spectrum of SHG in the $1^{\text {st }}$ order of periodical poling (shown in Fig.1) demonstrated an excellent agreement with expected characteristics of the periodical poling. Fig. 2 shows SHG spectrum in the $1 / 2$-order of periodical poling with a maximum SHG efficiency at $675 \mathrm{~nm}$. The inset demonstrates the square dependence of the SHG power on pump power. The demonstrated 'red' shift of SHG generation in the $1 / 2$-order paves the way for a substantial expansion of the tuning range of the visible periodically-poled diode-pumped laser sources.

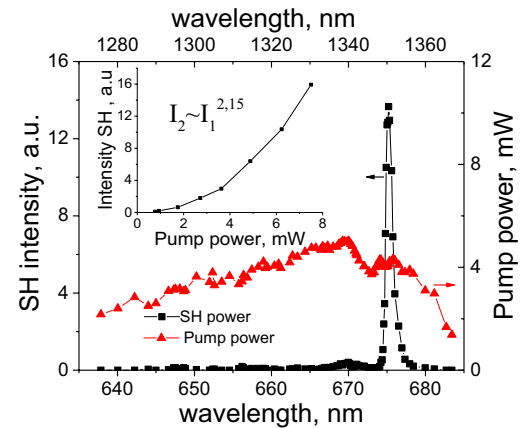

Fig.2. SHG power in $1 / 2$-order of periodical poling (black squares) and pump power (red triangles) vs wavelength. Inset shows SHG power vs pump power.

In summary, we demonstrated second harmonic generation in a periodically-poled lithium niobate crystal with a $1 / 2$-order of periodical poling. The utilization of the fractional poling period made it possible to extend the tuning range of the second harmonc generation to the long-wavelength range.

\section{ACKNOWLEDGMENT}

This research was supported by Russian Foundation for Basic Research, project no. 17-02-00865.

\section{REFERENCES}

[1] A. Kaltenbach, J. Hofmann, D. Seidel et al., Proc.SPIE 10088, 1008808 (2017).

[2] M.M. Fejer, G.A. Magel, D.H. Jundt et al., IEEE J. of Quant. El. 28, 26312654 (1992).

[3] K.A. Fedorova, G.S. Sokolovskii, D.A.Livshits et al., Opt. Lett. 39, 66726674 (2014).

[4] K.A. Fedorova, G.S. Sokolovskii, P.R. Battle et al., Opt. Lett., 40, 835838 (2015).

[5] E.G.Sokolovskaya, K.A.Fedorova, V.V.Dudelev et al., J. of Phys.: Conf. Ser. 769, 012050 (2016). 